SLAC-PUB-10420

astro-ph/0403608

March 2004

\title{
The $J$-band Light Curve of SN 2003lw, Associated with GRB 031203
}

\author{
A. Gal-Yam ${ }^{1,2}$, D.-S. Moon ${ }^{1,3}$, D. B. Fox ${ }^{1}$, A. M. Soderberg ${ }^{1}$, S. R. Kulkarni ${ }^{1}$, E. Berger ${ }^{1}$, \\ S. B. Cenko ${ }^{1}$, S. Yost ${ }^{1}$, D. A. Frail ${ }^{4}$, M. Sako ${ }^{5}$, W. L. Freedman ${ }^{6}$, S. E. Persson ${ }^{6}$, P. \\ Wyatt $^{6}$, D. C. Murphy ${ }^{6}$, M. M. Phillips ${ }^{7}$, N. B. Suntzeff ${ }^{8}$, P. A. Mazzali ${ }^{9,10}$ K. Nomoto ${ }^{10}$ \\ avishay@astro.caltech.edu
}

\begin{abstract}
At $z=0.1055$, the gamma-ray burst GRB 031203 is one of the two nearest GRBs known. Using observations from the Very Large Array (VLA) and Chandra $X$-ray Observatory we derive sub-arcsecond localizations of the radio and X-ray afterglow of this GRB. We present near-infrared observations of the supernova SN 2003lw, which exploded in the host galaxy of the GRB 031203. Our deep, high resolution Magellan/PANIC data establish that this SN is spatially coincident with the radio and X-ray localizations of the afterglow of GRB 031203 to subarcsecond precision, and is thus firmly associated with the GRB. We use image differencing to subtract the bright emission from the host galaxy, and measure the time evolution of the SN between $\sim 5$ and $\sim 50$ days after the GRB. The resulting light curve has a shape which is quite different from that of the two SNe previously associated with GRBs, SN 1998bw and SN 2003dh. With SN
\end{abstract}

\footnotetext{
*Work supported in part by the Department of Energy Contract DE-AC03-76SF00515

${ }^{1}$ Division of Physics, Mathematics, \& Astronomy, MS 105-24, California Institute of Technology, Pasadena, CA 91125

${ }^{2}$ Hubble Fellow

${ }^{3}$ Robert A. Millikan Fellow

${ }^{4}$ National Radio Astronomy Observatory, P.O. Box 0, Socorro, New Mexico 87801

${ }^{5}$ Stanford Linear Accelerator Center, 2575 Sand Hill Road M/S 29, Menlo Park, CA 94025

${ }^{6}$ Observatories of the Carnegie Institution of Washington, 813 Santa Barbara Street, Pasadena, CA 91101

${ }^{7}$ Las Campanas Observatory, Carnegie Observatories, Casilla 601, La Serena, Chile

${ }^{8}$ Cerro Tololo Inter-American Observatory, National Optical Astronomy Observatory, Casilla 603, La Serena, Chile

${ }^{9}$ INAF, Osservatorio Astronomico di Trieste, Via Tiepolo, 11, I-34131 Trieste, Italy

${ }^{10}$ Dept. of Astronomy and RESCEU, University of Tokyo, Tokyo 113-0033, Japan
} 
2003lw securely associated with this burst, we confirm that all three GRBs with redshifts $z<0.3$ were accompanied by SN explosions.

Subject headings: supernovae: individual (SN 2003lw) - gamma rays: bursts

\section{Introduction}

The emerging association between long-duration gamma-ray bursts (GRBs) and type Ic supernovae (SNe Ic) is perhaps the most significant breakthrough in our understanding of GRBs, and may also provide new insights into the physics of core-collapse SNe and the deaths of massive stars. The initial strong evidence for this connection came from the spatial and temporal coincidence between GRB 980425 and SN 1998bw (Galama et al. 1998). More recently, this picture was convincingly affirmed by the detection of SN features in the optical afterglow spectrum of GRB 030329 (Stanek et al. 2003; Hjorth et al. 2003; Matheson et al. 2003). Both of these milestone discoveries resulted from the study of the nearest GRBs yet identified, GRB 980425 at $z=0.0085$ and GRB 030329 at $z=0.1685$. Indeed, each relatively rare occurrence of a GRB at low redshift $(z<0.3)$ provides a unique opportunity for further study of the GRB-SN connection.

GRB $031203^{11}$ was detected by IBIS on board the INTEGRAL spacecraft on 2003 December 3, at 22:01:28 UT (Gotz et al. 2003). A fading X-ray afterglow was discovered by XMM-Newton on 2003 December 4 (Santos-Lleo et al. 2003; Campana et al. 2003; Rodriguez-Pascual et al. 2003) and found to be consistent with the location of a radio transient (Frail 2003; Soderberg, Kulkarni, \& Frail 2003). Prochaska et al. (2003a; 2003b; 2004) identified the host galaxy of the X-ray and radio transients, determined its redshift $(z=0.1055)$ and studied its properties in detail. Recently, several groups (Bersier et al. 2004; Tagliaferri et al. 2004a; Thomsen et al. 2004; Cobb et al. 2004) reported optical photometric and spectroscopic observations of this GRB, which apparently reveal the signatures of an associated SN (designated SN 2003lw; Tagliaferri et al. 2004b). In spite of the low redshift of this event, its low Galactic latitude (less than $5^{\circ}$ from the plane) and the resulting Galactic extinction $(E[B-V]=1.04$; Schlegel, Finkbeiner, \& Davis 1998), as well as its bright host galaxy, make the study of this event challenging.

\footnotetext{
${ }^{11}$ The possible classification of this event as an X-ray flash (XRF) was debated in recent literature (e.g., Prochaska et al. 2004; Watson et al. 2004; Thomsen et al. 2004). However, the high-energy observations presented by Sazonov, Lutovinov, \& Sunyaev (2004) conclusively show that this event does not fit any of the commonly used definitions of XRFs.
} 
To overcome these difficulties, we have undertaken near infrared (NIR) observations. The results of this effort are reported in this Letter. Coordinated radio and X-ray observations, which enabled us to probe the total energy output of this sub-energetic GRB, are reported elsewhere (Soderberg et al. 2004).

\section{Observations}

\section{1. $\quad$ Near Infrared imaging}

The location of the X-ray and radio transients associated with GRB 031203 was observed with Persson's Auxiliary Nasmyth Infrared Camera (PANIC; equipped with a $1024 \times 1024$ HgCdTe Hawaii I infrared focal plane array), mounted on the Baade (Magellan I) $6.5 \mathrm{~m}$ telescope, during 5 nights, in the $J(1.25 \mu)$ and $K_{s}(2.16 \mu)$ bands. Due to their lower sensitivity and poor temporal coverage, we did not detect SN 2003lw in our $K_{s}$-band data. We therefore report in this Letter only the analysis of our $J$-band observations. We obtained 18 or 36 dithered frames during four epochs, approximately 5, 7, 50, and 81 days after the GRB. A journal of the observations is given in Table 1. The integration time for each frame was $120 \mathrm{~s}$. We obtained a common sky frame for each data set from the stacked image cube, and subtracted it from each frame. We then carried out flat fielding, and shifted each frame to a common reference position to create the final image.

\subsection{Image subtraction and SN photometry}

In order to extract the faint SN signal from the bright host galaxy background, we have used the Common Point-spread-function (PSF) image subtraction method (CPM; Gal-Yam, Poznanski, \& Maoz, in preparation $)^{12}$. We find that extra flux is detected in our three early epochs (obtained 5.38, 7.39 and 50.21 days after the GRB) when compared with our latest epoch (day 81.11; see Fig. 1). We therefore proceed in our analysis by assuming that our latest image contains only a negligible amount of SN light. This is supported by J-band observations presented by Cobb et al. (2004).

\footnotetext{
${ }^{12} \mathrm{CPM}$ compares a new image with a reference one by extracting an empirical point-spread-function (PSF) from both images, and convolving each image with the PSF of the other. While slightly degrading the final seeing (as the final PSF of both images is worse than the initial PSF of either image), this procedure produces output images with nominally identical PSFs, while introducing a minimal amount of noise. In particular, we have found that this algorithm often produces difference images which have lower subtraction residuals near the nuclei of bright galaxies compared to other popular image subtraction packages, and thus allows
} 
To accurately measure the SN flux in each of the difference frames (Fig. 1, panels a-c), we have added an artificial point source, of known negative flux and with the native PSF, to our reference image. This results in the introduction of a positive artificial star to each of the difference images, which should have an identical PSF to any real variable point source (the native PSF of the reference image convolved with the PSF of the image from which it was subtracted). This source is visible in panels a-c of Fig. 1, in the lower left quadrant. Using aperture photometry (with the aperture equal to the FWHM of the artificial star), we measure the flux of SN 2003lw with respect to this constant source, and derive the relative photometry of SN 2003lw. We have identified 22 objects which appear in the 2MASS point source catalog and fall into the PANIC field of view. Of these, the brightest 6 are saturated or non-linear in our images. We have used the remaining 16 objects to tie our zero-point to the 2MASS photometry. We then used 10 secondary calibrators in the close vicinity of GRB 031203 to set the absolute calibration of the artificial reference star. From the scatter in calibrations derived using different secondary calibrators, we estimate a final absolute calibration error of $0.08 \mathrm{mag}$. Assuming this zero point, we calculate the $J$-band magnitudes of SN 2003lw at 5.38, 7.39 and 50.21 days after GRB 031203, which are reported in Table 2 and plotted in Fig. 3. Our calibration yields $J$-band magnitudes for the host galaxy and the SN which are consistent with those reported by Cobb et al. (2004).

The main source of error in the derivation of the magnitudes reported here is the uneven background levels of the difference images, caused by effects such as non-perfect flat-fielding and sky subtraction (as seen in panels a-c of Fig. 1). In order to quantify this error, we have added 20 artificial point sources to each of the images, at random positions sampling both empty sky and high-background areas of the image, with the appropriate PSF. We then subtracted the reference image using CPM. We photometered the resulting difference frames and have iteratively adjusted the flux of the artificial sources until their mean measured flux from the difference image was equal to the flux we measured for SN 2003lw at the same epoch. Finally, we adopted the measured scatter in the magnitudes of the artificial sources as the error in the SN magnitude at that epoch. We find that these errors are far larger than other sources of error, e.g., the Poisson errors in the aperture photometry of the bright artificial reference star we used in our photometric sequence, which are therefore neglected. To these errors we add in quadrature our absolute calibration uncertainty given above.

better sensitivity to variable sources which are superposed on bright galactic background. 
Table 1. J-band observations of SN 2003lw

\begin{tabular}{llll}
\hline \hline Epoch & \multicolumn{1}{c}{ UT date } & $\begin{array}{c}\text { Days after } \\
\text { GRB 031203 }\end{array}$ & $\begin{array}{l}\text { Number of } \\
\text { 120s frames }\end{array}$ \\
\hline 01 & 2003 December 9, 07:07:39 & 5.38 & 18 \\
02 & 2003 December 11, 07:20:05 & 7.39 & 18 \\
03 & 2004 January 23, 03:10:01 & 50.21 & $36 \times 2^{\mathrm{a}}$ \\
04 & 2004 February 23, 00:45:25 & 81.11 & 36 \\
\hline
\end{tabular}

${ }^{a}$ Data from two consecutive nights were combined.

Table 2. J-band photometry of SN 2003lw

\begin{tabular}{lll}
\hline \hline \multicolumn{1}{c}{ UT date } & \multicolumn{1}{c}{ Days after } & $J$ magnitude \\
& GRB 031203 & \\
\hline 2003 December 9, 07:07:39 & 5.38 & $21.66 \pm 0.60$ \\
2003 December 11, 07:20:05 & 7.39 & $20.57 \pm 0.21$ \\
2004 January 23, 03:10:01 & 50.21 & $20.60 \pm 0.21$ \\
\hline
\end{tabular}



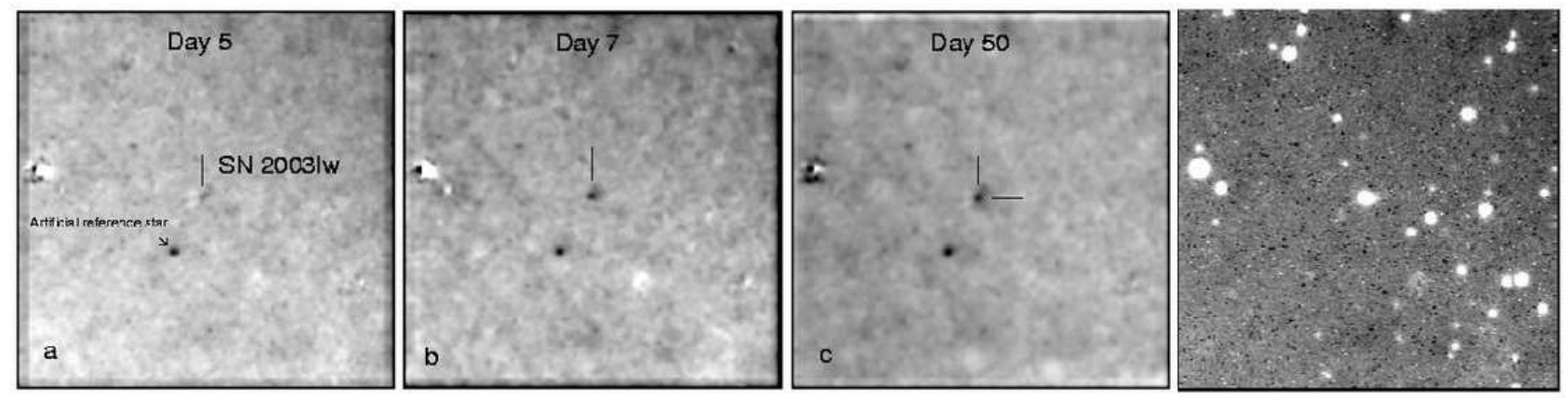

Fig. 1.- NIR detection of SN 2003lw. Panels a-c show 37.7" $\times 37.7$ " sections of the difference images obtained by subtracting our last observed epoch (81 days after the GRB) from the first three epochs, obtained at 5.38, 7.39 and 50.21 days post burst. SN 2003lw is at the center of each frame (indicated by crosshairs) and its brightness can be compared to an artificial reference star added to each image (marked in panel a) which has a constant flux. The Grey levels in panels a-c are linearly scaled between the peak of the artificial reference star and $1 \sigma$ of the sky noise below the mode sky level. Comparing panels $\mathrm{b}$ and $\mathrm{c}$, note the similar IR flux detected at 7 and 50 days after the GRB. For orientation purposes, panel d shows a section from our reference (fourth epoch) PANIC image with the same scale and orientation. The host galaxy of GRB 031203/SN 2003lw lies at the center of panel d. North is up, East to the left. 


\section{Results}

\subsection{Spatial Coincidence with the Radio and X-ray Afterglow of GRB 031203}

The position of the radio afterglow from VLA observations (Soderberg et al. 2004) is (J2000) RA 08:02:30.1833(18), Dec -39:51:03.522(78), as referenced to the ICRS (uncertainties in the final digits are given in parentheses). This position is derived from our first-epoch $22 \mathrm{GHz}$ observation, the highest resolution of our various radio observations, which had a beam size of $1.14 \times 0.29$ arcsec.

The position of the X-ray afterglow is derived from our Chandra X-ray Observatory observations. We observed the afterglow with Chandra in a single 21.6 ksec exposure beginning at 21:35 UT on 22 January 2004 (mean epoch 49.1 days post-burst), with the afterglow position at the aimpoint of the ACIS-S3 CCD. Data were reduced in the standard manner using the CIAO tools (v. 3.0.2). In particular, a comprehensive "wavdetect" source detection analysis reveals six X-ray sources on the S3 chip with coincident objects in the GSC-2.2 catalog. Using these sources we confirm the absolute astrometry to \pm 0.13 arcsec precision, and derive a position for the afterglow of (J2000) RA 08:02:30.159, Dec - 39:51:03.51, with 0.18" uncertainty. We then identify two X-ray sources on the S3 chip with point-like sources in our 9 December 2003 PANIC $J$-band image. By registering these two X-ray source positions against their $J$-band counterparts, we are able to locate the afterglow on the PANIC image to $\approx 1.2$-pixel precision.

The resulting X-ray localization, $0.56 \times 0.64$ arcsec in size, is shown as the red ellipse in Figure 2, along with the VLA radio (cyan) and PANIC NIR transient (green) localizations, as transferred to the same image. All ellipses are two-sigma (95\% confidence level). The WCS on the PANIC image is derived from the positions of 23 2MASS counterparts, and the uncertainty in the VLA position is dominated by the $0.095 \times 0.121$ arcsec RMS of this mapping. The NIR localization is derived from two of our subtracted images; the two relative localizations differ by $(0.23,0.46)$ pixels and so we adopt $0.029 \times 0.058$ arcsec as our one-sigma uncertainty.

Fig. 2 shows that the location of the NIR transient is consistent with that of the afterglow across all wavelengths, to high precision, confirming the association that has already been inferred from the temporal coincidence of GRB 031203 and SN 2003lw.

Moreover, this location is consistent with the peak of the host galaxy light: the NIR offset, which has the highest precision, is $0.15 \pm 0.23$ pixels West, $0.26 \pm 0.46$ pixels North. At $z=0.1055$, this $0.04 \pm 0.06$ arcsec offset corresponds to a physical distance of $0.07 \pm 0.13 \mathrm{kpc}$. 


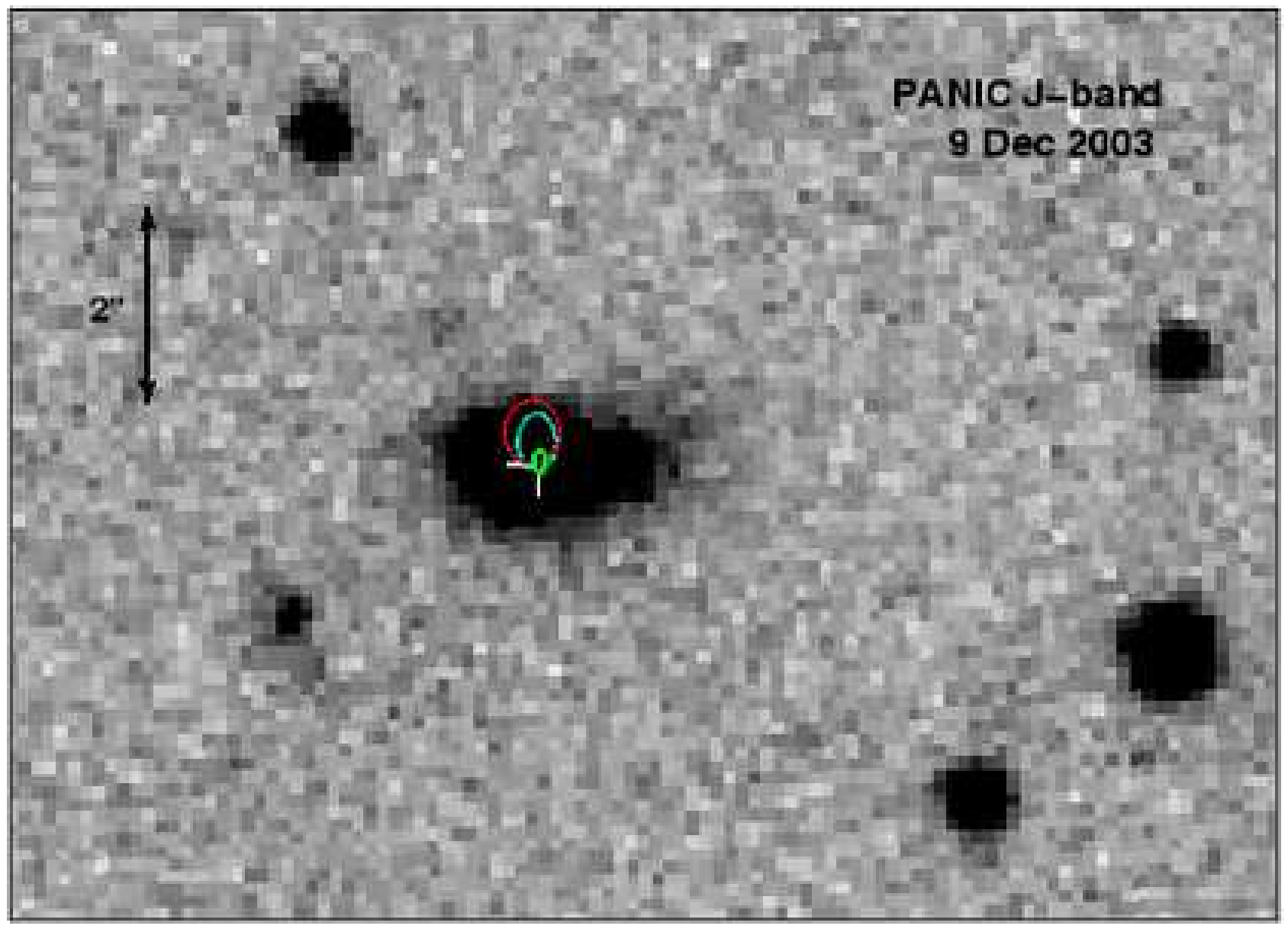

Fig. 2.- PANIC J-band image of the host galaxy of GRB 031203 from 9 December 2003, with localizations of the Chandra X-ray afterglow (red), the VLA radio afterglow (cyan), and the PANIC NIR transient (green) superposed; the position of the central maximum of the host galaxy light is indicated by the white ticks, and North is up and East is to the left. The ellipses shown are two-sigma (95\%-confidence) localizations; see text for details. The NIR transient ellipse is $0.46 \times 0.92$ pixels $(0.06 \times 0.11$ arcsec $)$ in size. The size of the VLA ellipse is $0.38 \times 0.58$ arcsec; the $\mathrm{X}$-ray ellipse is $0.56 \times 0.64$ arcsec. Although transient light contributes to this image, its effect on the appearance of the host galaxy at this color stretch is negligible. The localizations of the afterglow and transient are consistent across all wavelengths, and locate the source within $0.2 \mathrm{kpc}$ of the central maximum of the host galaxy light. 


\subsection{The J-band Light Curve}

Figure 3 shows the $J$-band light curve of SN 2003lw. Our deep observations detect the SN light as early as $\sim 5$ days after the burst (the earliest detection of this SN), and suggest a steep rise in flux between the first two epochs ( $\sim 5$ and $\sim 7$ days after the GRB). The flux measurements at $\sim 7$ and $\sim 50$ days after the GRB are comparable.

To better interpret these observations, we wish to compare our data to the $J$-band light curve of SN 1998bw, the prototype and best observed GRB-associated SN. Unfortunately, NIR observations of SN 1998bw are scarce - the only data available are 3 epochs of photometry and spectroscopy reported by Patat et al. (2001). We have used these NIR spectra, in conjunction with optical spectroscopy from the same source, to calculate the $I-J$ colors of SN 1998bw, redshifted to $z=0.1055$, using the methods described by Poznanski et al. (2002). We then construct a model for the J-band light curve of SN 1998bw as it would appear at $z=0.1055$ in the following manner. Based on the observations of Galama et al. (1998) we construct the I-band light curve of SN 1998bw at $z=0.1055$, using the extinction correction given by Cobb et al. (2004). The resulting curve is shown in Fig. 3 (dashed curve). We then use the three epochs of $I-J$ colors calculated above, along with the appropriate (smaller) J-band extinction correction (from Prochaska et al. 2004) to calculate $J$-band anchors for our light curve model (asterisks in Fig. 3). Finally, we assume that the shapes of the $J$ and $I$-band light curves of SN 1998bw are similar, and adjust the $I$-band light curve upward by the mean of the three color measurements we have, yielding the solid curve plotted in Fig. 3. Note that using the $I$-band light curve shape we can obtain a decent fit to all three $J$-band anchors, and thus it appears that assuming this light curve shape is reasonable, at least during the declining part of the light curve, sampled by the available NIR photometry of SN 1998bw.

Having exhausted the relevant observational data, we turn to models of SN 1998bw. Iwamoto et al. (1998) present hydrodynamical models of exploding C+O stars which reproduce the optical light curve and spectra of SN 1998bw. Synthetic spectra calculated by these authors extend out to $1.2 \mu$. We use these spectra to calculate synthetic $I-J$ colors of SN 1998bw (as it would seem at $z=0.1055$ ) between $\sim 10$ and $\sim 107$ days after the GRB, as we have done with the real date. Errors resulting from incomplete coverage of the $J$-band by the available spectra, are calculated as in Poznanski et al. (2002). We then repeat the process described above, creating a new set of synthetic anchors (marked by small filled circles in Fig. 3). The distribution of these anchor points suggests again that the $I$ and $J$-band light curves of SN 1998bw had similar shapes. Indeed, applying the average synthetic color correction $I-J \sim 0.9$ and the appropriate extinction correction to our model $I$-band light curve of SN 1998bw, the resulting model $J$-band light curve of SN 1998bw (light solid curve 
in Fig. 3) fits the synthetic anchors very well. The $J$ band model light curves derived from the sparse NIR observations (boldsolid curve) and the model spectra (light solid curve) are reasonably consistent with each other and support the notion that the $I$ and $J$-band light curves of SN 1998bw had similar shape. Interestingly, the rough $I-J$ colors of SN 2003lw measured by Cobb et al. (2004; $I-J \sim 1$ ) appear similar to the colors we derive for SN $1998 \mathrm{bw}$ at $z=0.1055$.

Comparing our model SN 1998bw light curves with the data, an obvious discrepancy is immediately revealed. While our late-time point is marginally consistent with the models, the early-time $J$-band data points fall significantly below model expectations (by at least $\sim 0.9 \mathrm{mag}$, a $9 \sigma$ effect). This discrepancy is enhanced if SN 2003lw was intrinsically brighter than SN 1998bw by $\sim 0.4 \mathrm{mag}$, as advocated by Thomsen et al. (2004). We also note that the fast early rise implied by our early data points is not reproduced by either model. Making SN 2003lw less luminous overall would make the model inconsistent with our late time data point, and would conflict with the observations of Thomsen et al. (2004) and Cobb et al. (2004). Thus, it appears that SN 2003lw had a significantly different light curve shape than SN 1998bw, at least in the $J$-band.

\section{Discussion and Conclusions}

In this Letter, we have presented $J$-band observations of SN 20031w. The sensitivity and resolution of our data enabled us to pinpoint the location of SN 2003lw within its host galaxy, and to show it is consistent with sub-arcsecond localizations of the radio and X-ray afterglow of GRB 031203, thus confirming the association of these two events. The precise NIR localization of this event also puts it within $0.2 \mathrm{kpc}$ from the host galaxy center. The $J$-band light curve of SN 2003lw shows a rapid initial rise ( $5-7$ days after the GRB) and evidence for bright emission more than 50 days after the GRB. The fast early rise of SN 2003dh, associated with GRB 030329, has been interpreted by Woosley \& Heger (2003) and Mazzali et al. (2003) as evidence for asymmetry in the explosion. A thorough investigation of this possibility will probably require an analysis of our data in combination with other extensive data sets of optical and NIR photometry and spectroscopy collected by other groups (e.g., Thomsen et al. 2004; Tagliaferri et al. 2004; Bersier et al. 2004; Cobb et al. 2004).

Cobb et al (2004) have recently reported $I$ and $J$-band observations of this event, obtained with the SMARTS $1.3 \mathrm{~m}$ telescope. A direct comparison between our observations and the SMARTS data, obtained on numerous epochs, is complicated by the fact that these authors do not present the light curve of SN 20031w. Instead, they plot the temporal evolution of the combined light of the SN and its bright host galaxy, derived from aperture 


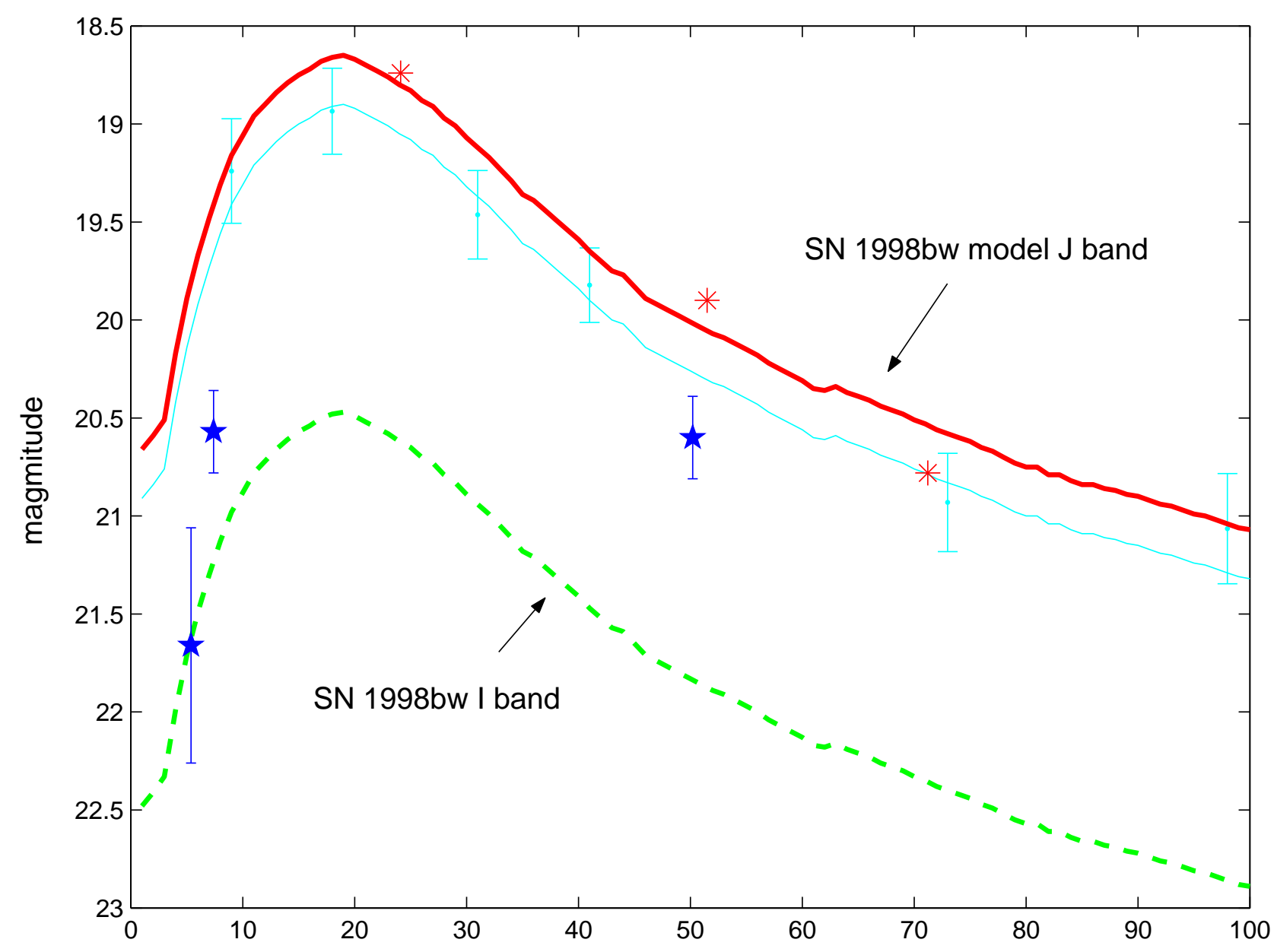

Fig. 3.- J-band photometry of SN 2003lw. Our measurements (Stars with $2 \sigma$ error bars, see text) are compared to model light curves of SN 1998bw. The bold dashed line shows the $I$-band light curve of SN 1998bw, redshifted to $z=0.1055$, assuming an extinction of $A_{I}=1.4 \mathrm{mag}$ (Cobb et al. 2004). The only available $J$-band spectra of SN 1998bw (Patat et al. 2001) are used to calculate $I-J$ color offsets, correcting for the different extinction values in the $I$ and $J$ bands (from Prochaska et al. 2004; see text). These points are plotted as asterisks, and serve as our guidance in constructing a model $J$-band light curve for SN 1998bw. This is done by adopting the I-band light curve shape, normalized to fit these three points (bold solid curve). A second model, based on $J$-band points calculated using synthetic color corrections (small circles, see text) is shown as a light solid curve. Note that both $J$-band models are inconsistent with our early data points. 
photometry, which shows considerable scatter. It is thus hard to say whether their data show the same early fast rise we detect. Our observation that SN 2003lw had similar flux levels 7 and 50 days after the GRB is consistent with the reported SMARTS data. Furthermore, comparing their I-band data with model light curves of SN $1998 \mathrm{bw}$, these authors arrive at the conclusion that the light curve shape of SN 2003lw does not resemble that of SN 1998bw. This is in accord with our analysis of the $J$-band data (Cobb et al. 2004 do not attempt to compare their $J$-band data with a model of SN 1998bw). It appears that both our observations and the Cobb et al. (2004) data set suggest that SN 2003lw had a light curve quite unlike that of SN 1998bw; with a fast rise to maximum, which appears broader, and perhaps showing a secondary peak in the IR.

The above discussion provides further evidence for the diversity of SNe associated with GRBs [see, e.g., Thomsen et al. (2004) and Lipkin et al. (2004) for recent reviews]. With SN 2003lw, all three nearby GRBs are firmly associated with SNe, apparently supporting suggestions made, e.g., by Podsiadlowski et al (2004), that all long GRBs are accompanied by SNe. It may well be the case that the focus of future studies should now move from proving the association between SNe and GRBs to an attempt to characterize the properties of this population of SNe. Such studies may provide new clues about the progenitors and engines of GRBs, by requiring viable GRB models to be able to produce the large quantities of nickel derived from the SN observations, as well as valuable insights into possible explosion mechanisms of core-collapse SNe, which may involve GRB-like aspherical effects [e.g., Khokhlov et al. (1999), see Gal-Yam et al. (2004) for further discussion]. Low-redshift GRBs, expected to be localized in larger numbers by the upcoming SWIFT mission, as well as systematic studies of local core-collapse SNe (e.g., Berger et al. 2003; Soderberg et al. 2004, in preparation; Stockdale et al. 2004) will probably shed more light on these intriguing questions.

\section{Acknowledgments}

We thank D. Poznanski for his help with synthetic photometry. A.G. acknowledges support by NASA through Hubble Fellowship grant \#HST-HF-01158.01-A awarded by STScI, which is operated by AURA, Inc., for NASA, under contract NAS 5-26555. The Guide Star Catalog was produced at the Space Telescope Science Institute under U.S. Government grant. These data are based on photographic data obtained using the Oschin Schmidt Telescope on Palomar Mountain and the UK Schmidt Telescope. 


\section{REFERENCES}

Berger, E., Kulkarni, S. R., Frail, D. A., \& Soderberg, A. M. 2003, ApJ, 599, 408

Bersier, D., et al. 2004, GRB Circular Network, 2544, 1

Campana, S., Tagliaferri, G., Chincarini, G., Covino, S., Fugazza, D., \& Stella, L. 2003, GRB Circular Network, 2478, 1

Cobb, B. E., Bailyn, C. D., van Dokkum, P. G., Buxton, M. M., \& Bloom, J. S. 2004, ApJL, submitted, ArXiv Astrophysics e-prints, astro-ph/0403510

Frail, D. A. 2003, GRB Circular Network, 2473, 1

Galama, T. J., et al. 1998, Nature, 395, 670

Gal-Yam, A., Poznanski, D., Maoz, D., Filippenko, A. V., \& Foley, R. J. 2004, PASP, submitted, ArXiv Astrophysics e-prints, astro-ph/0403296

Gotz, D., Mereghetti, S., Beck, M., Borkowski, J., \& Mowlavi, N. 2003, GRB Circular Network, 2459, 1

Hjorth, J., et al. 2003, Nature, 423, 847

Iwamoto, K., et al. 1998, Nature, 395, 672

Khokhlov, A. M., Höflich, P. A., Oran, E. S., Wheeler, J. C., Wang, L., \& Chtchelkanova, A. Y. 1999, ApJ, 524, L107

Lipkin, Y. M. et al. 2003, ApJ, in press, ArXiv Astrophysics e-prints, astro-ph/0312594

Matheson, T., et al. 2003, ApJ, 599, 394

Mazzali, P. A., et al. 2003, ApJ, 599, L95

Patat, F., et al. 2001, ApJ, 555, 900

Podsiadlowski, P., Mazzali, P. A., Nomoto, K., Lazzati, D., \& Cappellaro, E. 2004, ApJL, submitted, ArXiv Astrophysics e-prints, astro-ph/0403399

Poznanski, D., Gal-Yam, A., Maoz, D., Filippenko, A. V., Leonard, D. C., \& Matheson, T. 2002, PASP, 114, 833

Prochaska, J. X., Chen, H. W., Hurley, K., Bloom, J. S., Graham, J. R., \& Vacca, W. D. 2003a, GRB Circular Network, 2475, 1 
Prochaska, J. X., Bloom, J. S., Chen, H. W., Hurley, K., Dressler, A., \& Osip, D. 2003b, GRB Circular Network, 2482, 1

Prochaska, J. X., et al. 2004, ApJ, submitted, ArXiv Astrophysics e-prints, astro-ph/0402085

Rodriguez-Pascual, P., Santos-Lleo, M., Gonzalez-Riestra, R., Schartel, N., \& Altieri, B. 2003, GRB Circular Network, 2477, 1

Sazonov, S. Yu., Lutovinov, A. A., \& Sunyaev, R. A., in prep.

Santos-Lleo, M., Calderon, P., \& Gotz, D. 2003, GRB Circular Network, 2464, 1

Schlegel, D. J., Finkbeiner, D. P., \& Davis, M. 1998, ApJ, 500, 525

Soderberg, A. M., Kulkarni, S. R., \& Frail, D. A. 2003, GRB Circular Network, 2483, 1

Soderberg, A. M., et al. 2004, in prep.

Stanek, K. Z., et al. 2003, ApJ, 591, L17

Stockdale, C. J., Van Dyk, S. D., Sramek, R. A., Weiler, K. W., Panagia, N., Rupen, M. P., \& Paczynski, B. 2004, IAU Circ., 8282, 2

Tagliaferri, G., et al. 2004a, GRB Circular Network, 2545, 1

Tagliaferri, G., et al. 2004b, IAU Circular 8303

Thomsen, B., et al. 2004, A\&A, submitted, ArXiv Astrophysics e-prints, astro-ph/0403451

Watson, D., et al. 2004, ApJL, submitted, ArXiv Astrophysics e-prints, astro-ph/0401225

Woosley, S. E. \& Heger, A. 2003, ApJ, submitted, ArXiv Astrophysics e-prints, astro$\mathrm{ph} / 0309165$ 